It will also cover the more interesting case of terminal blending at the end of a trajectory where useful information can be found from the earlier part of the trajectory.

The outstanding difficulties to be confronted in pursuit of the general line of attack above are as follows: (1) Single vaporizing-droplet meteoroids appear to occur in the size range accessible only to radar observation. (2) Meteors following the two-stage ablation model (melting and spraying, followed by vaporization of droplets) occur from one extreme, in which the meteoroid is exhausted at a much smaller atmospheric pressure than are the droplets, through one in which the meteoroid is exhausted at about half the pressure at which the last droplets are spent, to one in which the droplets are largely vaporized over a rise in pressure that is small compared with the pressure at the end point. (a) Only one meteor fits the first extreme at present and it is one observed by radar and television. (b) Many of the Super-Schmidt meteors may fall in the intermediate category. (c) A few Super-Schmidt meteors are in the last category.The best possible classification and study of the observations is important here. (3) Larger meteoroids have additional problems caused by viscous flow of the meteoroid in air and pooling of wakes of droplets. (4) For great fireballs this regime passes over to the very complicated one of a detached bow shock ahead of the meteoroid and a boundary layer feeding into the wake. (5) The role of thermal shock should be evaluated. The relatively slow time scale for the aerodynamic flux of heat followed by relaxation of any thermal stresses by melting makes this mechanism unlikely to play an important role in meteors except in the case where a local structural failure suddenly exposes a still-cold surface to the air stream. In such a case, indeed, progressive fracturing, exposing still more material, could lead to a rapidly propagating collapse of the entire meteoroid into dust. (6) Finally, there is the problem of the apparently low crushing strength of many meteoroids. It is possible that such a meteoroid undergoes varying bending while yawing or rotating such that resolution along principal axes of the stress tensor indicates tension in one direction; this results in a significant fraction of the aerodynamic load appearing as a rapidly varying tensile load (R. E. McCrosky, private communication).

\title{
REFERENCE
}

Boitnott, C. A., Savage, H. F. $\quad$ 1972, Astrophys. Journ., 172, 201.

\section{METEOR ORBITS}

\section{Kresák}

As the final stage of reductions of small-scale photographs recording irregular interactions of meteoroids with the atmosphere, the determination of meteor orbits remains a difficult task with regard to both the extent of the data and their precision. Among recent contributions to the lists of photographic meteor orbits, the greatest share comes from the Harvard Super-Schmidt Program, 1956-59. The number of fireball orbits from the Prairie Network has increased to 235, including the Lost City meteorite of 4 January 1970, the second fall (after Prríbram) for which a photographic orbit is available (05.105.081). The same reference lists eight meteors that, according to their photometric and flight parameters, may be high-density objects; this sample is characterized by low geocentric velocities, low inclinations, and aphelia far inside the orbit of Jupiter. Results from the European All-Sky Network also include several cases of meteorite-like objects, but no find has been reported. The occurrence of three fireballs with low extinction level (Bramberg, $36 \mathrm{~km}$; Buedingen, $31 \mathrm{~km}$; Marburg, $32 \mathrm{~km}$ ) over the German stations during two consecutive nights, 21 and 22 March 1972, is interesting. The orbits computed by Ceplecha and Ježková do not suggest any association among these objects.

On the other end of the mass spectrum, long-term cosmic-dust experiments, including the determination of velocity vectors by multiple-coincidence acoustic sensors, were made on Pioneer 8 and Pioneer 9 spacecrafts. Orbital elements of 16 particles of micron size computed by Berg and Richardson reveal an astonishing abundance of very short periphelion distances $(<0.1 \mathrm{AU})$ and revolution periods of less than $1 \mathrm{yr}$; two orbits are nearly identical with that of the Earth, three are hyperbolic, and only one is similar to those of short-period comets. Two meteoroid velocity meas- 
urements on the secondary satellite launched with the Scout OFO vehicle (Johnson et al., 1971) suggest that these may possibly be lunar ejecta caused by the Gemini shower. More accurate measurements are urgently needed to verify these anomalies, unprecedented in the use of other observing techniques sensitive to other mass ranges.

The difference between the distribution of the orbits of fireballs and of faint photographic meteors is much less pronounced but much better established. There is a definite displacement of the aphelia of short-period fireballs from the orbit of Jupiter to the asteroid belt (McCrosky, 1968). The displacement is opposite that required for a mass separation by the Poynting-Robertson effect. The other striking difference between the Prairie Network fireballs and fainter meteors, a lack of long periods and high inclinations in the former group, may be explained by observational selection (03.104.001).

The selection problems are also of great importance in the interpretation of the radar meteor orbits. According to the recent results of Southworth (Coll. 13; personal communication), recombination at low altitudes and an extremely steep ionization velocity dependence produce very strong biases against low-velocity objects. If one accounts for these losses in observation, it appears that at the Earth's distance from the Sun we are imbedded in a cloud of nearly circular orbits. The corrections would bring the radar data much closer to the Pioneer measurements as regards the revolution periods; on the other hand, both the photographic and the radar show a distinct deficiency of periphelion distances of less than $0 \cdot 1 \mathrm{AU}$ and present no evidence for lunar ejecta.

The uncertainty in the determination of the velocity vectors, mainly due to the extrapolation of atmospheric deceleration, puts an upper limit on the accuracy of the periods and aphelion distances. This limit is unfortunately too low for applying perturbational computations to individual meteoroids in order to trace their past orbital evolution by the methods that proved very useful for comets and asteroids. The unknown nongravitational effects represent an additional prohibitive factor. On the other hand, the data are adequate for a statistical treatment, and the existence of meteor streams as families of particles of common origin distributed randomly over large volumes of space makes them an excellent object for statistical investigation of the perturbations. Even the methods of secular perturbations, the feasibility of which has been seriously questioned in the case of comets, are of value for the problems of meteor-stream dispersion. Such methods were applied by Galibina (Symp. 45) and Zausaev (Symp. 45) to study the secular evolution of the Leonid, Quadrantid, and $\delta$ Aquarid streams during a time span of $4000 \mathrm{yr}$. Selected meteors of the $\alpha$ Virginid and $\alpha$ Capricornid streams were followed over two centuries by numerical integrations of Kazimirchak-Polonskaya et al. (Symp. 45). Model computations by Levin et al. (Symp. 45) on a short-period stream meeting Jupiter show the waving and dispersal of individual filaments and confirm that the local perturbations at close encounters may broaden a stream much more effectively than does the accumulated effect of differential secular variations. By another model computation, Delcourt (Symp. 45) finds the Poynting-Robertson effect unimportant for a period of $20000 \mathrm{yr}$ even at subvisual magnitudes. However, other nongravitational effects have to be considered before relying on the models of evolution based on purely gravitational interactions.

A special kind of problem is posed by meteor swarms of recent origin, which have not yet had enough time to disperse into a continuous ring around the orbital ellipse. Sekanina (Coll. 22) used the orbital and apparition data of such swarms for reconstructing the conditions under which they may have been formed by cometary ejections. Thorough analyses, including the ejection velocities and radiation effects, were made by McIntosh (Coll. 13; 1972) for the two richest meteor showers of this century, the Leonids and the Draconids. The failure of the prediction of a rich Draconid display in 1972 is a good illustration of the incompleteness of the data obtained from Earth-bound observations. It appears that the knowledge of the motion of the parent comet combined with the one-dimensional information from a few passages through the stream is generally insufficient for obtaining a clear insight into the history of the stream and predicting its future activity at various distances from the comet.

Another important evolutionary effect, not limited to meteor streams, is the growth of the perturbations in a nearly resonant motion with Jupiter. This situation does not allow an application of 
Monte Carlo methods or of methods of secular perturbations. The resonances may produce significant irregularities in the smooth distribution of orbital elements and induce librating motions. The distribution of the revolution periods of all available photographic meteor orbits has been searched by Lindblad (Coll. 13) for the presence of resonance gaps. The result was positive, which points to a minor importance of the dynamical nongravitational effects. Unfortunately, even for the best-determined orbits the probable errors are still comparable with the width of the gaps.

It is obvious that the present population of interplanetary particles has originated from larger bodies late in the history of the solar system. The exposure ages of chondrite meteorites are of the order of $10^{7} \mathrm{yr}$. There is no method of comparable accuracy for determining ages of the smaller particles that disintegrate in the atmosphere, but in all probability their lifetimes, controlled by erosion effects, do not exceed $10^{4}-10^{5} \mathrm{yr}$; otherwise, there would be a much smaller proportion of shower meteors and comet-meteor relationships than actually observed. At a state of quasi-equilibrium, the general distribution of the orbits of the parent bodies should have been essentially the same as the contemporary one, so that the problems of orbital evolution are divided into two areas: (1) the history of the sizable parent bodies, and (2) the independent history of the meteoroids broken off from them. The radiation ages and survival times controlled by the rate of collisions and erosion admit only those processes by which the fragments can be transported fast enough from the initial orbit into an Earth-crossing orbit.

In the orbital evolution of the potential parent bodies, the gravitational effects of Jupiter clearly predominate over those of all other planets. As long as the nongravitational effects can be ignored, the potential evolutionary tracks in the phase space of orbital elements closely follow the constant values of the Jacobian integral in the restricted three-body problem. The Jacobi constant was shown by Kresák (Coll. 13; 1972) to represent not only an indicator of the evolution but also a significant dividing line between the comets and the asteroids. The only indefinite region is that of the librations, and even here we have a good secondary criterion in the stability of the librating motion. Three kinds of processes, perturbations at close encounters with the inner planets, nongravitational accelerations by cometary ejections, and nondestructive collisions, can make the objects deviate from the Jacobian tracks; for some of these effects the direction of the deviation can be established. Information on the probable sources can be obtained from the orbit in which the meteoroids meets the Earth, and some evidence of correlated nonuniformities in both the rate of occurrence and some atmospheric characteristics of the meteors is found.

With computer programming, the Monte Carlo methods for integrating perturbations at close encounters, as suggested by Öpik, developed by Arnold, and refined by Wetherill and Anders, have become a very important tool. They have a great advantage in their ability to yield the time scale of the variations and the relative probability of individual types of final orbits. As the exposure ages are well established for most types of meteorites and can be used as an independent check of the theory, meteorites have become the main objective of these investigations. The lack of good orbits of the meteorites (there are only two, Přibram and Lost City) is moderated by the availability of some secondary statistical data, such as the diurnal variation of meteorite falls, and by the increasing information on the orbits of very bright fireballs.

Wetherill (Coll. 12) applied Monte Carlo calculations to a wide variety of potential cometary, asteroidal, and lunar sources of meteorites and compared the resulting distribution of Earthcrossing orbits with the low-velocity component of the Prairie Network flux. He found that only initial orbits with periphelion near the Earth, aphelion near Jupiter ( $\sim 4.5 \mathrm{AU})$ and low inclination would be satisfactory. Calculated exposure ages for such a source agree excellently with the observed values for the chondrites. Wetherill (Coll. 13) interprets this result as evidence of the cometary origin of most meteorites. According to his computations for the asteroidal sources, the transport from Mars-crossing to Earth-crossing orbits is much too slow, and collisional destruction will eliminate essentially all the products of these collisions before encounter with the Earth. The Apollo objects, with collision lifetimes of the order of $10^{8} \mathrm{yr}$, remain the only alternative source compatblie with these calculations.

On the other hand, Anders (1972) suggests that metorites come from the asteroid belt. He assumes 
that the low exposure ages are controlled by the destruction lifetimes, which are of just the right order of magnitude according to Dohnanyi $(1972 ;$ Coll. 13). The difference in the exposure ages of different classes of meteorites is related to their different crushing strength, as pointed out by Mazor et al. (04.105.011). This interpretation requires either a much more intense source to maintain the observed flux in the vicinity of the Earth, or a much more effective perturbing mechanism, or both. The latter may perhaps result from the existence of the resonance surfaces in $a, e^{\prime}, i^{\prime}$ space, inducing large oscillations in inclination and eccentricity, and hence also in periphelion distance (Williams, Coll. 12). Anders argues that most meteorites have originated from six to eleven parent bodies with radii between 100 and $300 \mathrm{~km}$, which are probably related to about seven Mars-crossing or Marsapproaching asteroid families.

The observed diurnal variation of meteorite falls requires that they strike the Earth during the first few passes, if at all. This may be due to either a quick elimination by encounters with Jupiter near the aphelion (Wetherill, cometary source) or to a quick collisional destruction in the asteroid belt (Anders and Dohnanyi, asteroidal source). In fact, the Lost City meteorite, with a radiation age of $5 \times 10^{6} \mathrm{yr}$, did not intersect the orbit of the Earth for much of the past $5 \times 10^{5} \mathrm{yr}$, according to Lowrey (05.105.080).

Thus the fundamental question as to the proportion of cometary and asteroidal meteoroids in different size ranges still remains open. There is little doubt about the asteroidal origin of the highly differentiated types of meteorites, achondrites, and irons, and about the cometary origin of the dustball meteors and meteor streams with aphelia near or beyond Jupiter. A cometary origin for the majority of other objects appears very plausible, as demonstrated by Wetherill, if a suitable mechanism can reduce the aphelion distances to about $4 \mathrm{AU}$. In fact, $P /$ Encke is the only known comet with $Q<4 \cdot 5$, and as a unique object it appears to have contributed very significantly to the present meteor flux (Whipple, 1967). The existence of compact meteor streams of still much smaller aphelion distance, such as the Geminids, suggests that there are powerful nongravitational effects acting even on the sizable parent bodies. The critical phase of evolution, bringing the aphelion far enough inside the orbit of Jupiter, favors nearly circular precapture orbit with high values of the Jacobian integral, similar to that of $P$ /Oterma before its encounter with Jupiter in 1937.

When speaking about 'cometary' and 'asteroidal' meteoroids, one must consider another problem of vital importance: What is the dividing line between comets and asteroids, and do some objects pass this line as they evolve? Some comets have almost asteroidal appearance (especially a few librating comets, according to Marsden, 03.102.003, Coll. 12), and it has been repeatedly suggested that some of the asteroids (especially Hidalgo and the nearly spherical Apollo objects like Alinda or Icarus) are virtually extinct comet nuclei, exhausted of volatiles. In this case, some meteoroids may have originated from parent bodies that are now classified as asteroids but that at the time of separation were normal comets producing gaseous envelopes and ejecting meteoric matter. There is little doubt that a number of objects of this kind revolve in the inner part of the solar system without being discovered. Sekanina (1972) has detected in radar data some minor streams that may be associated with Apollo- and Albert-type bodies.

Quite analogously, various types of meteorites seem to be of different origin and orbital history, reflecting much more than different layers within some uniform type of parent body. Each sample of meteor orbits obtained by a particular technique is probably a mixture of different types of objects, in unknown proportion. This is best illustrated by the results of the Prairie Network and the European All-Sky Network, where an unexpectedly low rate of recoveries combines with an unexpectedly high flux of bright fireballs, often much brighter than the objects recovered as meteorites.

\section{REFERENCES}

Dohnanyi, J. S. 1972, Icarus, 17, in press.

Johnson, J. H., Farmer, B. J., King, B. R., Bagwell, R. G., McAllum, W. E. Proc. XIVth COSPAR Plenary Mtg., Seattle, 1971, in press.

Kresák, L. $\quad$ 1972, Bull. Astron. Inst. Czech., 23, 1. 
McCrosky, R. E. $\quad$ 1968, in L. Kresák and P. M. Millman (eds.), Proc. IAU Symp. 33, 265.

McIntosh, B. A., Jr. 1972 , Roy. Astron. Soc. Canada, 66, 149.

Sekanina, Z. $\quad 1972$, Icarus, 17, in press.

Whipple, F. L. 1967, in J. L. Weinberg (ed.), The Zodiacal Light and the Interplanetary Medium, NASA

SP-150, 409.

METEOR SHOWERS

B. A. Lindblad

Photographic, Radar, and Theoretical Studies

In the period 1969-72, several lists of northern-hemisphere, photographic, and radar meteor streams have been published. Lindblad (06.104.042) has listed 75 photographic stream radiants. Cook et al. (1973) have further analyzed the photographic data. Cook (Coll.13) gives a list of stream radiants and orbital elements. Sekanina $(05.104 .009,10)$ has discussed streams detected in the Harvard Radio Meteor data. Several new southern-hemisphere radar streams and associations have been reported by Gartrell (1971). The so-called 'toroidal' type of meteor stream (short period, high inclination, low eccentricity) is confirmed in the southern-hemisphere radar data.

Progress in identifying new streams has been made mainly through the use of computer techniques. The $D$-criterion of Southworth and Hawkins directly compares different sets of orbital elements and is easily adapted for computer searches. This method has been used by Gartrell (ibid.), Lindblad (06.104.076, 7), and Sekanina (op. cit.). A search by Lindblad among 2401 graphically reduced Super-Schmidt orbits revealed 15 new photographic streams for which identifications with visual radiants were proposed. Several streams were split into a northern and a southern branch. The so-called $\sigma$ Leonid stream appears to be identical with the visual Virginid stream reported by Hoffmeister.

The precise photographic-data samples now available have made possible a detailed study of the orbital scatter within meteor streams. For meteor streams of short period this problem has been discussed by Lindblad (1972; Coll. 22). A progressive increase in the scatter of the orbital elements within a given stream is found as the mean period is reduced, indicating that the spatial motions of individual stream members differ increasingly from the average motion of stream members as the short-period streams drift inward toward the Sun. A systematic increase in mean meteoroid density with decreasing period is also noted.

The age of meteor streams has been discussed by Astapovich (1972). The determination of radiant positions and the radiation area of meteor streams was made by Chebotarev (06.104.009). Structural peculiarities and perturbations in the Perseid stream have been investigated by Kramer and Musiy (02.104.055) and by Kramer and Pavlenko (06.104.008). The evolution of a hypothetical meteor stream under the influence of Jupiter and Saturn has been studied by Sherbaum (06.104.027, 33). The orbital elements of photographic Leonids have been reported by Babadzhanov and Getman (03.104.022). Radar observations of the 1957-68 Leonids have been studied by McIntosh and Millman (03.104.029).

Comet-meteor stream associations. The problem of ejection from a parent comet and the early evolution of a meteor stream have been studied by Sekanina (1972, see reference, preceding Section). Lists of theoretical radiant positions derived from comet orbits have been published by Zentsev (03.102.019) and Kramer (06.104.048). The relation of Comet Halley to the Orionids and $\eta$ Aquarids has been discussed by Hajduk (03.014.005). Comet-stream associations have been discussed by Lindblad (op. cit.). The relation between the Scorpionid stream and Comet $1770 \mathrm{I}$ has been discussed by Bakharev (03.104.049). Several other authors have reported meteor streams that may be associated with Comet Lexell 1770 I. Some inherent difficulties of the suggested associations with this comet have been pointed out by Cook et al. (1973). Associations between comet orbits and radar meteor streams have been studied by Sekanina (op. cit.) and Gartrell (op. cit.). The PoyntingRobertson effect and its influence on particles ejected from Comet $1866 \mathrm{I}$ is discussed by Sherbaum and Shaido (06.104.028). 